\title{
Ventilação Mecânica Não-Invasiva Aplicada em Pacientes com Insuficiência Respiratória Aguda após Extubação Traqueal*
}

\author{
Noninvasive Positive Pressure Ventilation in Patients with \\ Acute Respiratory Failure after Tracheal Extubation
}

\author{
Anderson José1, Luiz Rogério de Carvalho Oliveira², Elaine Cristina Polleti Dias ${ }^{3}$, Daniela Brandão Fuin ${ }^{4}$, \\ Leslie Gomes Leite4, Graziele de Souza Guerra ${ }^{4}$, Deise do Carmo Barbosa ${ }^{4}$, Paulo Antonio Chiavone 5 .
}

\section{RESUMO}

JUSTIFICATIVA E OBJETIVOS: A ventilação mecânica não-invasiva (VMNI) tem sido utilizada rotineiramente como método para auxiliar o desmame da ventilação mecânica. Uma das aplicações mais comuns é a sua utilização em pacientes que evoluem com quadro de insuficiência respiratória aguda $(\operatorname{IRpA})$ após a extubação traqueal, embora as evidências científicas para esta indicação ainda sejam controversas. Os objetivos deste estudo foram identificar o número de pacientes que evoluem para IRpA após a extubação, avaliar a eficácia da VMNI para reverter este quadro e promover

1. Fisioterapeuta Especialista em Fisioterapia em Pneumologia e Fisioterapia Respiratória, Supervisor do Curso de Fisioterapia Pneumo-Funcional e Fisioterapia Intensiva da Santa Casa de São Paulo, Professor do Curso de Fisioterapia da Universidade Nove de Julho (UNINOVE).

2. Fisioterapeuta Especialista em Fisioterapia em Pneumologia e Fisioterapia Respiratória, Supervisor do Curso de Fisioterapia Pneumo-Funcional e Fisioterapia Intensiva da Santa Casa de São Paulo, Fisioterapeuta do Hospital Paulistano.

3. Fisioterapeuta Especialista em Fisioterapia Cardiorrespiratória, Supervisora do Curso de Fisioterapia Pneumo-Funcional e Fisioterapia Intensiva da Santa Casa de São Paulo.

4. Fisioterapeuta Especialista em Fisioterapia Pneumo-Funcional.

5. Doutor em Medicina, Professor Assistente da Faculdade de Ciências Médicas da Santa Casa de São Paulo, Diretor do Serviço de Terapia Intensiva da Santa Casa de Paulo.

*Recebido da Irmandade da Santa Casa de Misericórdia de São Paulo - Serviço de Terapia Intensiva, São Paulo, SP

Apresentado em 20 de setembro de 2006

Aceito para publicação em 07 de novembro de 2006

Endereço para correspondência:

Anderson José

Rua Porto Sabaúna, 266

03206-000 São Paulo, SP

Fone: (11) 6912-4142 ou 9572-7241

E-mail: dr_andersonjose@yahoo.com.br

(C)Associação de Medicina Intensiva Brasileira, 2006 aumento da taxa de sucesso no desmame da ventilação mecânica.

MÉTODO: Foi realizado um estudo prospectivo e transversal. A VMNI foi aplicada nos pacientes que apresentaram IRpA após extubação, independentemente de sua etiologia. A VMNI foi realizada na modalidade pressão de suporte, que foi ajustada para se obter volume-corrente exalado (Vte) de 6 a $8 \mathrm{~mL} / \mathrm{kg}$ e PEEP e $\mathrm{FiO}_{2}$ ajustados para se obter a $\mathrm{SaO}_{2}>95 \%$. A VMNI foi realizada de forma contínua até cessarem os sinais de IRpA apresentados. O sucesso do desmame e da VMNI foi definido quando os eventos que levaram o paciente à utilização da VMNI fossem revertidos por um período superior a 48 horas de ventilação espontânea, evitando assim a re-intubação.

RESULTADOS: Foram incluídos no estudo 103 pacientes. Observou-se que 32\% (33) evoluíram com sinais de IRpA após a extubação e foram submetidos a VMNI. O tempo de VMNI utilizado foi $8 \pm 5$ horas, a PSV utilizada foi de $12 \pm 2 \mathrm{cmH}_{2} \mathrm{O}$, PEEP de $7 \pm 2 \mathrm{cmH}_{2} \mathrm{O}, \mathrm{FiO}_{2}$ de $40 \% \pm 20 \%$, Vte de $462 \pm 100 \mathrm{~mL}$, FR de $26 \pm 5 \mathrm{rpm}$. Entre os pacientes submetidos a VMNI (33), 76\% (25) cursaram com sucesso e posterior alta do serviço de terapia intensiva e $24 \%$ (8) evoluíram com insucesso da VMNI e necessidade de re-intubação.

CONCLUSÕES: A VMNI aplicada em pacientes com IRpA após a extubação foi um recurso seguro e eficaz para evitar a re-intubação.

Unitermos: desmame, insuficiência respiratória aguda após a extubação, ventilação mecânica não-invasiva

\section{SUMMARY}

BACKGROUND AND OBJECTIVES: Noninvasive positive pressure ventilation (NPPV) has been routinely used to assist the weaning of the mechanical ventilation. One of the applications most common is in patients who had acute respiratory failure after extuba- 
tion, even the scientific evidences for this indication still controversy. The aims of this study were to evaluate the index of patients that evolve for respiratory failure after extubation and evaluated the effectiveness of NPPV to avoid the need for reintubation and to promote increase in success index of weaning.

METHODS: We conducted a transversal and prospective study. It was applied to NPPV in the patients who presented respiratory failure after extubation, independent of its etiology. NPPV was applied in to pressure support ventilation, with Vte for 6 to $8 \mathrm{~mL} / \mathrm{kg}$, PEEP and $\mathrm{FiO}_{2}$ adjusted to reach $\mathrm{SaO}_{2}>95 \%$. The NPPV was accomplished of a continuous mould even interrupt the signs of respiratory failure presented initially. The success of weaning and the NPPV was defined when the clinical events were reverted by a period greater than 48 hours in spontaneous breathing, avoid thus reintubation.

RESULTS: We included 103 patients. Noted that 32\% (33) evolved with signals of respiratory failure after extubation and were submitted to NPPV. The time of NPPV was on mean $8 \pm 5$ hours, PSV of $12 \pm 2 \mathrm{cmH}_{2} \mathrm{O}$, PEEP of $7 \pm 2 \mathrm{cmH}_{2} \mathrm{O}, \mathrm{FiO}_{2}$ of $40 \% \pm 20 \%$, Vte of $462 \pm 100$ $\mathrm{mL}$, RR of $26 \pm 5 \mathrm{rpm}$. Among patients who accomplished NPPV (33), 76\% (25) attended with success and them afterwards let the ICU. Of the patients assigned to NPPV, $24 \%$ (8) did not tolerate the procedure and were reintubated.

CONCLUSIONS: We conclude that NPPV is safe and effective in averting the need for reintubation in patients with respiratory failure after extubation.

Key Words: acute respiratory failure after extubation, noninvasive positive pressure ventilation, weaning

\section{INTRODUÇÃO}

A ventilação mecânica não-invasiva (VMNI) vêm sendo rotineiramente utilizada em alguns serviços como um método para auxiliar o desmame da ventilação mecânica. Uma das aplicações mais comuns é a sua utilização em pacientes que evoluem com quadro de insuficiência respiratória aguda (IRpA) após a extubação traqueal, embora as evidências científicas para esta indicação ainda sejam controversas.

Os primeiros estudos da aplicação da VMNI no auxílio do desmame são referentes ao seu uso em pacientes com extubação acidental ou nos casos de IRpA após a extubação, como uma tentativa de evitar uma provável re-intubação ${ }^{1-6}$. Os resultados da aplicação da VMNI com este objetivo, diminuindo as taxas de insucesso no desmame são promissores. Este recurso foi recomendado pela Conferência Internacional de Consenso em Medicina Intensiva, como uma técnica indicada para prevenir a re-intubação ${ }^{7}$, entretanto, foi contra-indicado pelo relatório prévio do III Consenso Brasileiro de Ventilação Mecânicå. Dada a controvérsia e a alta aplicabilidade clínica desta situação, nota-se a necessidade de buscar maiores evidências para indicação da VMNI.

O objetivo deste estudo foi identificar o número de pacientes que evoluem com IRpA após a extubação traqueal, avaliar a eficácia da VMNI em reverter este quadro, evitar a re-intubação e promover um aumento nas taxas de sucesso no desmame da ventilação mecânica.

\section{MÉTODO}

Após a aprovação do projeto de pesquisa pelo Comitê de Ética em Pesquisa da Instituição, foi realizado um estudo prospectivo, observacional e transversal no Serviço de Terapia Intensiva da Irmandade da Santa Casa de Misericórdia de São Paulo.

Foram incluídos no estudo os pacientes que permaneceram sob ventilação mecânica por um período superior a 24 horas e submetidos ao processo de desmame da ventilação mecânica, de acordo com o protocolo de desmame adotado pelo serviço ${ }^{9,10}$, que apresentaram quadro de IRpA pós-extubação, diagnosticada através da presença de hipóxia com gasometria arterial apresentando $\mathrm{PaO}_{2}<60 \mathrm{mmHg}$ com o paciente em ar ambiente $\left(\mathrm{FiO}_{2}=0,21\right)$ e apresentando três ou mais dos sinais de IRpA: $\mathrm{PaCO}_{2}>$ $50 \mathrm{mmHg}, \mathrm{pH}<7,35$, Vte $<5 \mathrm{~mL} / \mathrm{kg}, \mathrm{FC}>120 \mathrm{bpm}$, $\mathrm{SaO}_{2}<90 \%, \mathrm{FR}>30 \mathrm{rpm}$ e presença de sinais clínicos de IRpA, como tiragens, uso de musculatura respiratória acessória, batimento de "asas" do nariz, cianose, dispnéia, padrão paradoxal e sudorese (Tabela 1).

\begin{tabular}{ll}
\multicolumn{2}{l}{ Tabela 1 - Critérios para Uso da VMNI e para Re-Intubação } \\
\hline Critérios para o Uso da VMNI & Critérios para Re-Intubação \\
\hline $\mathrm{PaO}_{2}<60 \mathrm{mmHg}$ & $\mathrm{PaO}_{2}<50 \mathrm{mmHg}$ \\
$\mathrm{PaCO}_{2}>50 \mathrm{mmHg}$ & $\mathrm{PaCO}_{2}>60 \mathrm{mmHg}$ \\
$\mathrm{pH}<7,35$ & $\mathrm{pH}<7,3$ \\
$\mathrm{Vte}<5 \mathrm{~mL} / \mathrm{kg}$ & $\mathrm{Vte}<4 \mathrm{~mL} / \mathrm{kg}$ \\
$\mathrm{FC}>120 \mathrm{bpm}$ & $\mathrm{FC}<60$ ou $>140 \mathrm{bpm}$ \\
$\mathrm{SaO}$ & $<90 \%$ \\
$\mathrm{FR}>30 \mathrm{rpm}$ & $\mathrm{SaO}_{2}<85 \%$ \\
\hline
\end{tabular}

VMNI = ventilação mecânica não-invasiva; FR = freqüência respiratória; Vte = volume corrente exalado. 
Foram excluídos do estudo, retornando para a ventilação mecânica invasiva, os pacientes que apresentaram instabilidade hemodinâmica, angina instável, necessidade de intubação para proteção das vias aéreas, trauma de face, rebaixamento do nível de consciência, escala de coma de Glasgow $\leq 8$, sangramento gastrintestinal ativo, pneumotórax não drenado, pós-operatório recente do trato digestivo alto, necessidade de sedação e pacientes com extubação traqueal acidental ou fora do protocolo estabelecido.

A VMNI foi realizada nos mesmos ventiladores mecânicos microprocessados que os pacientes estavam utilizando antes da extubação (Bird 6400, Bird 8400, Evita-S, Evita 2, Savina e Bennet 7200); foram utilizadas máscaras faciais de silicone e realizada constante monitorização respiratória e cardíaca. A modalidade ventilatória utilizada foi a pressão suporte - pressure support ventilation (PSV) - ajustada para se obter volume-corrente entre 6 e $8 \mathrm{~mL} / \mathrm{kg}$; a PEEP e a $\mathrm{FiO}_{2}$ utilizadas foram ajustadas para se obter $\mathrm{SaO}_{2}>95 \%$. A VMNI foi realizada de forma contínua até cessarem os sinais de IRpA apresentados pelo pacientes.

Foi considerado sucesso da VMNI e do desmame da ventilação mecânica quando o paciente permanecesse por um período superior a 48 horas em ventilação espontânea sem sinais de IRpA. Foi considerada falha da VMNI, sendo interrompida com conseqüente re-intubação dos pacientes, quando apresentaram hipoxemia diagnosticada através da presença de gasometria arterial apresentando a $\mathrm{PaO}_{2}<50 \mathrm{mmHg}$ com o paciente em ar ambiente $\left(\mathrm{FiO}_{2}=0,21\right)$ e a presença de três ou mais dos seguintes sinais: $\mathrm{PaCO}_{2}>60 \mathrm{mmHg}, \mathrm{pH}<$ 7,3 , Vte $<4 \mathrm{~mL} / \mathrm{kg}, \mathrm{FC}<60$ ou $>140 \mathrm{bpm}, \mathrm{SaO}_{2}<85 \%$, $\mathrm{FR}<10$ ou $>40 \mathrm{rpm}$ e a presença de sinais clínicos de IRpA (Tabela 1).

A análise estatística realizada compreendeu os testes $t$ de Student, t pareado, Mann-Withney, Exato de Fisher e cálculos de média e desvio-padrão.

\section{RESULTADOS}

Foram incluídos no estudo 103 pacientes. Desenvolveram IRpA após a extubação $32 \%$ (33) dos pacientes extubados e foram submetidos à VMNI. O diagnóstico destes 33 pacientes foi composto por $60 \%$ (20) de casos cirúrgicos e $40 \%$ (13) de casos clínicos. Cinqüenta e cinco por cento (18) dos pacientes eram do sexo masculino. A média de idade foi $46 \pm 20$ anos; o tempo de ventilação mecânica foi de $135 \pm 111$ horas; o tem- po de desmame da ventilação mecânica foi de $7 \pm 9$ horas; o APACHE II foi de $20 \pm 9$ pontos; as modalidades de desmame utilizadas foram a PSV em $82 \%$ (27) dos casos e o tubo T em 18\% (6) casos. Durante o início do desmame da ventilação mecânica, os pacientes apresentavam complacência estática de $45 \pm 20 \mathrm{~mL} /$ $\mathrm{cmH}_{2} \mathrm{O}$; resistência nas vias aéreas de $6 \pm 4 \mathrm{cmH}_{2} \mathrm{O} / \mathrm{L} /$ s; relação $\mathrm{PaO}_{2} / \mathrm{FiO}_{2}$ : $316 \pm 85$; freqüência respiratória de $20 \pm 5$ rpm; pressão inspiratória máxima (Pimáx): $-47 \pm 10 \mathrm{cmH}_{2} \mathrm{O}$; volume minuto (VE): $9 \pm 3$ litros; volume-corrente exalado (Vte): $485 \pm 186 \mathrm{~mL}$; relação f/Vt: $41 \pm 19 ; \mathrm{SpO}_{2}: 97 \pm 1$ (Tabela 2).

Tabela 2 - Casuística

\begin{tabular}{|c|c|}
\hline \multicolumn{2}{|l|}{ Diagnóstico } \\
\hline Casos cirúrgicos & $60 \%(20)$ \\
\hline Casos clínicos & $40 \%(13)$ \\
\hline \multicolumn{2}{|l|}{ Sexo } \\
\hline Masculino & $55 \%(18)$ \\
\hline Feminino & $45 \%(15)$ \\
\hline \multicolumn{2}{|l|}{ Forma de desmame } \\
\hline PSV & $82 \%(27)$ \\
\hline Tubo T & $18 \%(6)$ \\
\hline Idade (anos) & $46 \pm 20$ \\
\hline Tempo VM (horas) & $135 \pm 111$ \\
\hline Tempo de desmame (horas) & $7 \pm 9$ \\
\hline APACHE II (pontos) & $20 \pm 9$ \\
\hline $\mathrm{PaO}_{2} / \mathrm{FiO}_{2}(\mathrm{mmHg})$ & $316 \pm 85$ \\
\hline Cest $\left(\mathrm{mL} / \mathrm{cmH}_{2} \mathrm{O}\right)$ & $45 \pm 20$ \\
\hline Resistência $\left(\underline{\mathrm{cmH}}_{2} \underline{\mathrm{O}} / \mathrm{L} / \mathrm{m}\right)$ & $6 \pm 4$ \\
\hline Freqüência respiratória (ipm) & $20 \pm 5$ \\
\hline Pimáx $\left(\mathrm{cmH}_{2} \mathrm{O}\right)$ & $-47 \pm 10$ \\
\hline Volume minuto (litros) & $9 \pm 3$ \\
\hline Volume-corrente (mL) & $485 \pm 186$ \\
\hline Relação f/Vt & $41 \pm 19$ \\
\hline $\mathrm{SpO}_{2}$ & $97 \pm 1$ \\
\hline
\end{tabular}

Tempo VM = tempo total de ventilação mecânica; APACHE II = Physiology and Chronic Health Evaluation II; Cest = complacência estática; Pimáx = pressão inspiratória máxima; relação f/Vt = relação entre freqüência respiratória e volume corrente; $\mathrm{SpO}_{2}=$ oxi-saturação de pulso da hemoglobina.

A análise da gasometria arterial dos 33 pacientes submetidos a VMNI durante o período de desmame, após a extubação e durante a VMNI, respectivamente, encontra-se na tabela 3.

Considerando o total de pacientes estudados $(\mathrm{n}=$ 103), o sucesso do desmame da ventilação mecânica imediato, sem o auxílio da VMNI, foi de 68\% (70) dos pacientes; o sucesso do desmame auxiliado pela VMNI foi de $24 \%$ (25) dos pacientes, resultando em sucesso total do desmame da ventilação mecânica de 92\% (95) pacientes. Todos os que obtiveram sucesso do desmame evoluíram com alta do Serviço de Terapia Intensiva. 
Tabela 3 - Análise dos Gases Arteriais ( $n=33$ )

\begin{tabular}{lccc}
\hline $\begin{array}{l}\text { Gasometria } \\
\text { Arterial }\end{array}$ & $\begin{array}{c}\text { Durante o Desmame } \\
\text { (antes da extubação) }\end{array}$ & $\begin{array}{c}\text { Após a Extubação } \\
\text { (em ar ambiente) }\end{array}$ & $\begin{array}{c}\text { Durante a VMNI } \\
\mathrm{pH}\end{array}$ \\
$\mathrm{PaCO}_{2}$ & $7,45 \pm 0,06$ & $7,40 \pm 0,08$ & $7,41 \pm 0,09$ \\
$\mathrm{HCO}^{3}$ & $30 \pm 6$ & $31 \pm 5$ & $32 \pm 3$ \\
$\mathrm{BE}$ & $21 \pm 4$ & $19 \pm 4$ & $20 \pm 4$ \\
$\mathrm{PaO}_{2}$ & $-2 \pm 4$ & $-2 \pm 6$ & $-3 \pm 6$ \\
$\mathrm{SaO}_{2}$ & $104 \pm 21$ & $50 \pm 10$ & $93 \pm 20$ \\
$\mathrm{PaO}_{2} / \mathrm{FiO}_{2}$ & $97 \pm 1$ & $89 \pm 7$ & $96 \pm 1$ \\
\hline
\end{tabular}

Portanto, a taxa de insucesso no desmame foi de $8 \%$ (8); estes pacientes, apesar da VMNI, foram re-intubados e cursaram com taxa de mortalidade de $4 \%$ (4) casos (Tabela 4).

Tabela 4 - Análise dos Resultados do Desmame da Ventilação Mecânica ( $\mathrm{n}=103)$

\begin{tabular}{lc}
\hline Sucesso do desmame imediato & $68 \%(70)$ \\
IRpA após a extubação & $32 \%(33)$ \\
Sucesso do desmame com o auxílio da VMNI & $24 \%(25)$ \\
Sucesso do desmame total & $92 \%(95)$ \\
Insucesso do desmame & $8 \%(8)$ \\
Óbito & $4 \%(4)$ \\
\hline
\end{tabular}

VMNI = ventilação mecânica não-invasiva.

Considerando os pacientes que apresentaram IRpA após a extubação e foram submetidos a VMNI ( $\mathrm{n}=$ 33), 76\% (25) apresentaram reversão da IRpA e sucesso no desmame, porém $24 \%$ (8) destes pacientes houve falha e necessitaram de re-intubação e retorno para a ventilação mecânica invasiva. A pressão de suporte da VMNI utilizada nestes pacientes foi em média de $12 \pm 2 \mathrm{cmH}_{2} \mathrm{O}$, a PEEP utilizada foi de $7 \pm$ $2 \mathrm{cmH}_{2} \mathrm{O}$, a $\mathrm{FiO}_{2}$ administrada foi de $40 \pm 20 \%$, o Vte realizado pelos pacientes durante a VMNI foi de $462 \pm$ $100 \mathrm{~mL}$ e a freqüência respiratória espontânea de 26 $\pm 5 \mathrm{rpm}$. O tempo médio \pm desvio padrão que estes pacientes necessitaram de VMNI foi de $8 \pm 5$ horas (Tabela 5).

Tabela 5 - Análise dos Resultados da VMNI $(n=33)$

\begin{tabular}{lc}
\hline Tempo de VMNI (horas) & $8 \pm 5$ \\
PSV durante a VMNI $\left(\mathrm{cmH}_{2} \mathrm{O}\right)$ & $12 \pm 2$ \\
$\mathrm{FIO}_{2}$ durante a VMNI $(\%)$ & $40 \pm 20$ \\
PEEP durante a VMNI $\left(\mathrm{cmH}_{2} \mathrm{O}\right)$ & $7 \pm 2$ \\
Vte $(\mathrm{mL})$ & $462 \pm 100$ \\
$\mathrm{FR}(\mathrm{rpm})$ & $26 \pm 5$ \\
Sucesso da VMNI & $76 \%(25)$ \\
Falha da VMNI e re-intubação & $24 \%(8)$ \\
\hline
\end{tabular}

VMNI = ventilação mecânica não-invasiva; FR = freqüência respiratória; Vte: volume corrente exalado.

\section{DISCUSSÃO}

No serviço em que foi realizado o estudo, tem-se buscado, através de diversas ferramentas (monitorização respiratória, monitorização dos gases arteriais e índices de oxigenação, elaboração e aplicação de protocolos de desmame) encontrar resultados superiores no desmame da ventilação mecânica ${ }^{9-11}$. Foi publicado recentemente ${ }^{10}$, um acompanhamento em longo prazo da aplicação de um protocolo de desmame, em que os resultados nas taxas de insucesso atingiram a porcentagem de $9 \%$ apenas. O presente estudo foi mais uma tentativa para se buscar melhores resultados quanto ao desmame da ventilação mecânica.

No presente estudo, encontrou-se uma taxa de falha no desmame da ventilação mecânica de $8 \%$, resultado similar a outro estudo ${ }^{10}$, e uma taxa de mortalidade de $4 \%$. Isto vem corroborar com a idéia de que o desmame da ventilação mecânica necessita de critérios cientificamente comprovados para a sua condução, e reforçar a necessidade de abandonar técnicas baseadas apenas na experiência da equipe envolvida no processo do desmame.

Assim como este trabalho, alguns estudos têm sido realizados na tentativa de avaliar a eficácia da VMNI como auxiliar no processo de desmame. Neste sentido, três possibilidades têm sido verificadas:

- A aplicação da VMNI de forma rotineira em todos os pacientes após a extubação traqueal;

- A aplicação da VMNI depois da retirada precoce da ventilação mecânica, sem percorrer as etapas tradicionais do processo de desmame ou em pacientes extubados com abrandamento dos critérios de desmame; - A aplicação da VMNI em pacientes que desenvolvem IRpA após a extubação. É sobre esta aplicação específica as considerações a seguir:

Tentar evitar a re-intubação e conseqüentemente, o retorno para a ventilação mecânica invasiva, em pacientes que desenvolvem IRpA após a extubação pa- 
rece, à primeira vista, ser uma indicação interessante para a VMNI. Embora, certamente, já seja aplicada de forma rotineira em diversas UTI, inclusive no serviço onde este estudo foi realizado essa indicação ainda permanece controversa em virtude do pequeno número de artigos publicados, principalmente estudos controlados. Esta carência de estudos controlados pode ser atribuída à dificuldade de se aprovar em Comitê de Ética em Pesquisa, um projeto que propõe não realizar a VMNI em um grupo de pacientes com IRpA que claramente necessitam de assistência ventilatória.

Um estudo com desenho e resultados semelhantes com o trabalho que ora se apresenta, foi desenvolvido por Munshi e col. ${ }^{12}$. Estes pesquisadores apresentaram um estudo não controlado em que a VMNI foi utilizada em 72 pacientes vítimas de trauma e que apresentaram IRpA após a extubação. A aplicação da VMNI conseguiu reverter o quadro de IRpA e evitar o retorno para a ventilação mecânica invasiva em $72 \%$ (52 casos) destes pacientes. Os resultados do presente estudo foram semelhantes, apresentando taxa de efetividade para a VMNI de $76 \%$.

Em estudo controlado desenvolvido por Hilbert e col. ${ }^{13}$, foram estudados 60 pacientes com DPOC que foram extubados e apresentaram IRpA hipercápnica. Estes pacientes foram divididos em dois grupos: um grupo recebeu a VMNI e o outro grupo recebeu tratamento convencional que incluiu oxigenioterapia e cuidados clínicos. Concluíram que a VMNI reduziu significativamente a necessidade de re-intubação, reduziu o tempo de internação na UTI e reduziu o tempo de ventilação mecânica para tratar a IRpA após extubação, porém não alterou significativamente a letalidade intra-hospitalar.

Keenan e col. ${ }^{14}$ pesquisaram 81 pacientes que apresentaram IRpA após extubação, divididos em dois grupos: um grupo recebeu a VMNI e outro recebeu tratamento convencional com oxigenioterapia. Ao contrário dos estudos já mencionados, a VMNI não promoveu alterações significativas entre os dois grupos quanto ao número de re-intubação, duração da internação na UTI, duração da internação hospitalar, duração da ven- tilação mecânica e mortalidade.

O estudo de maior impacto sobre este assunto, desenvolvido por Esteban e col. ${ }^{15}$ pesquisaram 221 pacientes em 37 centros de 8 países. Compararam a aplicação da VMNI sob a modalidade PSV com a terapêutica tradicional em pacientes que desenvolveram IRpA após a extubação. Seus resultados não mostraram diferença quanto às taxas de re-intubação entre os dois grupos, concluindo que a VMNI atrasa, mas não evita uma reintubação. Entretanto, alguns indicadores mostraram piores resultados nos pacientes que fizeram uso de VMNI: para estes pacientes foi encontrado de forma significativa, maior atraso na re-intubação e mortalidade na UTI. Outros achados, sem diferenças significativas, foram relatados: o grupo que foi submetido a VMNI evoluiu com forte tendência para maior mortalidade intra-hospitalar e tempo de permanência na UTI (Tabela 6).

O presente estudo mostrou que a maioria dos pacientes beneficiou-se do tratamento proposto, apresentando reversão do quadro de IRpA e evoluindo com alta do Serviço de Terapia Intensiva, e isto entra em conflito com os resultados do estudo já comentado. Talvez, esta divergência deva-se ao fato de não contar com um grupo controle para comparar o que aconteceria com os pacientes que apresentaram IRpA após a extubação se não fossem submetidos à VMNI. A reflexão imediata leva a crer que estes pacientes necessitariam retornar para a ventilação mecânica invasiva e estariam sujeitos à gama de complicações inerentes à essa, comprometendo a sua evolução.

Um dado que reflete o papel de relevância da VMNI no processo de desmame deste estudo é a taxa de insucesso global do desmame: se a VMNI não tivesse sido utilizada como parte do protocolo de desmame, esta taxa de falha subiria dos $8 \%$ encontrados no estudo para $32 \%$. Portanto, a VMNI foi a responsável direta pelo sucesso no desmame de $24 \%$ dos pacientes. Este resultado foi obtido após um pequeno período de VMNI: em média, os pacientes foram submetidos a apenas 8 horas de VMNI, com parâmetros ventilatórios relativamente baixos (Tabela 5).

Tabela 6 - Estudos Controlados e Aleatórios que Utilizaram VMNI na IRpA após a Extubação Traqueal

\begin{tabular}{|c|c|c|c|c|c|c|c|c|}
\hline Autores & $\mathrm{n}=$ & $\begin{array}{l}\text { Tipos de } \\
\text { Pacientes }\end{array}$ & $\begin{array}{c}\text { Sucesso } \\
\text { (grupo VMNI) }\end{array}$ & $\begin{array}{c}\text { Sucesso } \\
\text { (grupo sem VMNI) }\end{array}$ & $p$ & $\begin{array}{c}\text { Mortalidade } \\
\text { (grupo VMNI) }\end{array}$ & $\begin{array}{c}\text { Mortalidade } \\
\text { (grupo sem VMNI) }\end{array}$ & $p$ \\
\hline Hilbert G e col. & 60 & DPOC & $80 \%$ & $33 \%$ & $<0,001$ & $2 \%$ & $6 \%$ & NS \\
\hline Keenan SP e col. & 81 & Geral & $28 \%$ & $31 \%$ & NS & $31 \%$ & $31 \%$ & NS \\
\hline Esteban A e col. & 221 & Geral & $52 \%$ & $52 \%$ & NS & $25 \%$ & $14 \%$ & 0,048 \\
\hline
\end{tabular}

VMNI = ventilação mecânica não-invasiva; $n$ = número de pacientes estudados; sucesso = porcentagem dos pacientes que não necessitaram retornar para a ventilação mecânica invasiva; NS = resultados sem estatística significativa. 
Por isso, discorda-se parcialmente do relatório prévio do III Consenso Brasileiro de Ventilação Mecânica, que classificou como grau de recomendação "A" para que a VMNI não seja utilizada na IRpA após a extubação. Esta afirmação se baseia apenas nos resultados de um único estudo, desconsiderando todos os demais. Acredita-se que ainda não há resultados conclusivos sobre este assunto e que há necessidade de maiores estudos controlados para se estabelecer um grau de recomendação adequado para este tipo de aplicação da VMNI.

Estes resultados conflitantes, ainda não invalidam a utilização da VMNI nas diferentes condições citadas, mas chamam a atenção de que os bons resultados dependem de uma série de fatores, muito provavelmente ainda não conhecidos, mas julga-se importante destacar:

- Os resultados dependem da causa da IRpA, sendo diferentes de acordo com esta causa, por exemplo, melhores nos pacientes com DPOC;

- Por ser uma técnica com baixas taxas de complicações, a VMNI pode ser utilizada precocemente;

- A falha da VMNI, quando presente, deve ser reconhecida imediatamente, sem retardos na intubação, uma vez que tal atraso pode levar a uma maior mortalidade; - Equipamentos adequados e equipes qualificadas e bem treinadas são pontos fundamentais para o sucesso da VMNI, que, portanto, pode variar de serviço para serviço.

Diante do exposto, concluiu-se que a VMNI aplicada a pacientes que apresentaram IRpA após a extubação foi um recurso seguro e eficaz na maioria dos casos para a reversão do quadro e, conseqüentemente, evitar a re-intubação e aumentar a taxa de sucesso no desmame da ventilação mecânica. A revisão da literatura demonstrou que ainda há resultados conflitantes com relação a esta aplicação, ressaltando a necessidade de evidências mais consistentes para esta indicação.

\section{REFERÊNCIAS}

01. Schettino GPP, Barbas CSV, Amato MBP et al - Ventilação Não-Invasiva com Pressão Positiva, em: Carvalho CRR - Ventilação Mecânica. Avançado. São Paulo: Atheneu, 2000;381-395.

02. Duarte A, Schettino GPP, Oliveira PH et al - Suporte ventilatório não-invasivo com pressão positiva e suporte ventilatório mecânica domiciliar. Relatório do II Consenso Brasileiro de Ventilação Mecânica. J Pneumol, 2000;26:(Suppl2):S439-S446.

03. Sarmento GJV - Ventilação Mecânica Não-Invasiva, em: Sarmento GJV - Fisioterapia Respiratória no Paciente Crítico: Rotinas Cllínicas. Barueri: Manole, 2005;90-97.

04. Richard J, Carlucci A, Wysocki M et al - French multicenter survey: noinvasive versus conventional mechanical ventilation. Am J Respir Crit Care Med, 1999;159:A367.

05. Antonelli M, Conti G, Rocco M et al - A comparison of noninvasive positive-pressure ventilation and conventional mechanical ventilation in patients with acute respiratory failure. N Engl J Med, 1998;339:429-435.

06. Brochard L, Mancebo J, Wysocki M et al - Noninvasive ventilation for acute exacerbations of chronic obstructive pulmonary disease. $\mathrm{N}$ Engl J Med, 1995;333:817-822.

07. International Consensus Conferences in Intensive Care Medicine: noninvasive positive pressure ventilation in acute respiratory failure. Am J Respir Crit Care Med, 2001;163:283-291.

08. Schettino GPP ; Reis MA ; Galas F et al - Ventilação mecânica não invasiva com pressão positiva. Relatório do III Consenso Brasileiro de Ventilação Mecânica. www.amib.com.br, 2006.

09. Oliveira LRC, José A, Dias EC et al - Protocolo de desmame da ventilação mecânica: efeitos da sua utilização em uma Unidade de Terapia Intensiva. Um estudo controlado, prospectivo e randomizado. RBTI, 2002;14:22-32.

10. Oliveira LRC, José A, Dias EC et al - Padronização do desmame da ventilação mecânica em unidade de terapia intensiva: resultados após um ano. RBTI, 2006;18:131-136.

11. José A, Dias EC, Santos VLA et al - Valor preditivo dos gases arteriais e índices de oxigenação no desmame da ventilação mecânica. RBTI, 2001;13:50-57.

12. Munshi IA, DeHaven B, Kirton O et al - Reengineering respiratory support following extubation: avoidance of critical care unit costs. Chest, 1999;116:1025-1028.

13. Hilbert G, Gruson D, Portel L et al - Noninvasive pressure support ventilation in COPD patients with postextubation hypercapnic respiratory insufficiency. Eur Respir J, 1998;11:1349-1353.

14. Keenan SP, Powers C, McCormack DG et al - Noninvasive positive-pressure ventilation for postextubation respiratory distress: a randomized controlled trial. JAMA, 2002;287:3238-3244.

15. Esteban A, Frutos-Vivar F, Ferguson ND et al - Noninvasive positivepressure ventilation for respiratory failure after extubation. $N$ Engl J Med, 2004;350:2452-2460. 